\title{
Investigation of the stress state of the elastic semi-strip with a transverse crack
}

\author{
Victor Reut, Nataly Vaysfel'd, Zinaida Zhuravlova \\ Odessa I.I. Mechnikov National University, Faculty of mathematics, physics and informational technologies \\ reut@onu.edu.ua \\ vaysfeld@onu.edu.ua, bttp:/ /orcid.org/0000-0001-8082-2503 \\ z.zhuravlova@onu.edu.ua, bttp://orcid.org/0000-0002-3271-8864
}

\begin{abstract}
The stress state of the semi-strip with a transverse crack is investigated. The mechanical load is applied to the semi-strip's short edge. The initial problem is reduced to the one-dimensional vector boundaryvalue problem with the help of the semi-infinite Fourier transformation applied by the generalized scheme. The vector boundary-value problem is solved with the help of the matrix differential calculation apparatus and Green's matrix-function apparatus. The solving of the problem is reduced to the solving of the system of three singular integral equations (SSIE). First singular equation contains two fixed singularities, so the special method is used for the SSIE's solving. Stresss intensity factors (SIF) are calculated for the different crack's length.
\end{abstract}

KEYWORDS. semi-strip; transverse crack; Green's matrix-function; fixed singularities; system of singular integral equations.

\section{INTRODUCTION}

The investigation of crack path in a semi-strip is an actual problem for many engineering applications. The detailed bibliography of the cracks in the plain elasticity bodies is given in [1-4].

The problems for an infinite strip containing a transversal crack were considered in the following works. In [5] the stress intensity factors were investigates in the case of a central transversal crack. The elastostatic plane problem for an infinite strip was leaded to a singular integral equation in [6].

Different approaches were applied for stress estimation in infinite strips with cracks. SIFs were calculated for the infinite strip with a crack with the help of the energetic method in [7]. The crack's spreading in the infinity strip was considered in [8], [9]. The solution of the problem for a loaded crack in an infinite strip was obtained with the help of the method of three problems superposition in [10]. The formulae for the stress intensity coefficients around cracks and rigid inclusions in an infinite strip were given in [11]. The theory of potentials was applied by the following authors for the problems of infinite strips and cracks. The boundary value problem for an infinite elastic strip with a semi-infinite crack was reduced to a singular integral equation by using the single and double layer potentials in [12]. The procedure based on Muskhelishvili's complex potential formulation and the combining conformal mapping technique of the stress intensity factors calculation for an infinite crack in a strip was suggested in [13].

The problems of the cracks between two different materials were solved in the following works. The solution of the problem of the biomaterial strips with the cracks was constructed with a help of the Green's function in [14]. The plane 
problem for a crack on the interface between an elastic strip and an elastic half-plane made of another material was investigated in [15].

The problems of the strips with multiple cracks were studied in the following works. The boundary value problem of the strip with the periodical cracks was reduced to a complex mixed problem, which was solved by the eigenfunction expansion of variation method in [16]. The solving of the problem for an infinite strip containing multiple cracks perpendicular to its boundaries was reduced to a system of the singular integral equations in [17]. The problem of the stress intensity factors determining in an infinite elastic strip containing two coplanar Griffith cracks was reduced by the use of Fourier transforms to solving of a set of integral equations in [18].

\section{THE STATEMENT OF A PROBLEM}

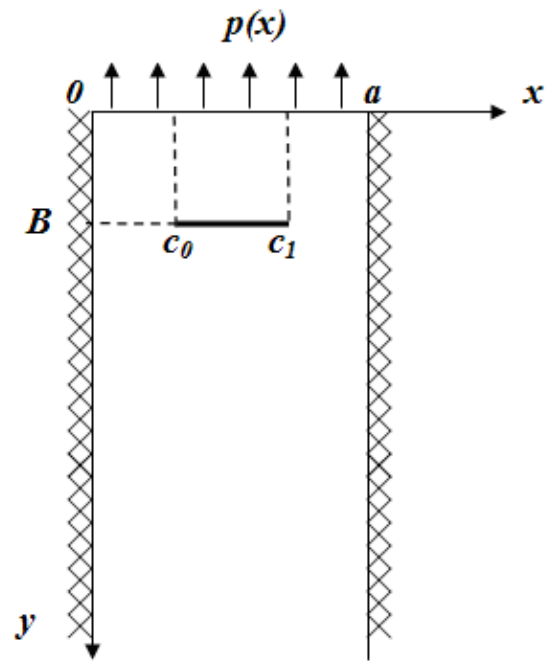

Figure 1: Geometry and the coordinate system of the semi-strip.

The elastic ( $G$ is a share module, $\mu$ is a Poison's coefficient) semi-strip (Fig. 1), $0<x<a, 0<y<\infty$ is considered under the mechanical load, which is applied along the semi-strip's short edge

$$
\sigma_{y}(x, 0)=p(x), \quad \tau_{x y}(x, 0)=0, \quad 0<x<a
$$

The lateral sides of the semi-strip are fixed

$$
u(0, y)=0, v(0, y)=0, u(a, y)=0, v(a, y)=0, \quad 0<y<\infty
$$

here $u(x, y)=u_{x}(x, y), v(x, y)=u_{y}(x, y)$ are the displacements satisfy the Lame's equations

$$
\left\{\begin{array}{l}
\frac{\partial^{2} u(x, y)}{\partial x^{2}}+\frac{\kappa-1}{\kappa+1} \frac{\partial^{2} u(x, y)}{\partial y^{2}}+\frac{2}{\kappa+1} \frac{\partial^{2} v(x, y)}{\partial x \partial y}=0 \\
\frac{\partial^{2} v(x, y)}{\partial x^{2}}+\frac{\kappa+1}{\kappa-1} \frac{\partial^{2} v(x, y)}{\partial y^{2}}-\frac{2}{\kappa-1} \frac{\partial^{2} u(x, y)}{\partial x \partial y}=0
\end{array}\right.
$$

where $\kappa=3-4 \mu$ is the Muskchelishvili constant.

Inside the semi-strip a transverse crack is located 


$$
\begin{aligned}
& \langle u(x, B)\rangle=\psi_{1}(x) \neq 0, \quad\langle v(x, B)\rangle=\psi_{2}(x) \neq 0, \quad c_{0}<x<c_{1} \\
& \left\langle\tau_{x y}(x, B)\right\rangle=0, \quad\left\langle\sigma_{y}(x, B)\right\rangle=0, \quad c_{0}<x<c_{1}
\end{aligned}
$$

here $\langle f(x, B)\rangle=f(x, B-0)-f(x, B+0)$.

One needs to solve the boundary value problem (1)-(4) to estimate the stress state of the semi-strip and SIF at the tips of the crack.

\section{THE GENERAL SOLVING SCHEME}

The initial problem (1)-(4) was reduced to the one-dimensional problem with the help of the semi-infinite sin-, cosFourier transformation applied by the generalized scheme [19].

Then the one-dimensional problem was reformulated in the vector form[20], [21]

$$
\begin{aligned}
& L_{2} \vec{y}_{\beta}(x)=\vec{f}(x) \\
& \vec{y}_{\beta}(0)=0, \vec{y}_{\beta}(a)=0
\end{aligned}
$$

where $L_{2} \vec{y}_{\beta}(x)=I_{y}{ }^{\prime \prime}(x)+2 \beta \vec{V}_{\beta}{ }^{\prime}(x)-\beta^{2} \overrightarrow{y y}_{\beta}(x), \vec{y}_{\beta}(x)=\left(\begin{array}{c}u_{\beta}(x) \\ v_{\beta}(x)\end{array}\right)$ is the vector of displacement

transformations, $\vec{f}(x)=\left(\begin{array}{c}\frac{3-\kappa}{\kappa+1} \chi^{\prime}(x)-\beta \sin \beta b \frac{\kappa-1}{\kappa+1} \psi_{1}(x)+\cos \beta b \frac{\kappa-3}{\kappa+1} \psi_{2}^{\prime}(x) \\ -\beta \frac{\kappa+1}{\kappa-1} \chi(x)-\sin \beta b \psi^{\prime}{ }_{1}(x)+\beta \frac{\kappa+1}{\kappa-1} \cos \beta b \psi_{2}(x)\end{array}\right), I$ is an identity matrix,

$P=\left(\begin{array}{cc}\frac{\kappa-1}{\kappa+1} & 0 \\ 0 & \frac{\kappa+1}{\kappa-1}\end{array}\right), Q=\left(\begin{array}{cc}0 & \frac{1}{\kappa+1} \\ -\frac{1}{\kappa-1} & 0\end{array}\right)$. Here the unknown function is inputted $\chi(x)=\left.v(x, y)\right|_{y=0}$.

The solution of the vector boundary problem (5) was obtained in the form [20]

$$
\vec{y}_{\beta}(x)=Y_{1}(x)\left(\begin{array}{l}
c_{1} \\
c_{2}
\end{array}\right)+Y_{2}(x)\left(\begin{array}{l}
c_{3} \\
c_{4}
\end{array}\right)+\int_{0}^{a} G(x, \xi) \vec{f}(\xi) d \xi
$$

where $Y_{1}(x), Y_{2}(x)$ are the fundamental matrix solutions, $c_{i}, i=\overline{1,4}$ are known constants, $G(x, \xi)$ is the Green's matrix function [20].

\section{THE SOLVING OF THE SINGULAR INTEGRAL EQUATIONS}

After inverting of the integral transformations the formulae for the displacements which contain three unknown functions $\chi^{\prime}(x), \psi_{1}^{\prime}(x), \psi_{2}^{\prime}(x)$ are obtained. The substitution of these formulae into conditions $\sigma_{y}(x, 0)=p(x), \tau_{x y}(x, B+0)=0, \sigma_{y}(x, B+0)=0$ reduces to the system of three singular integral equations: 


$$
\left\{\begin{array}{c}
\int_{-1}^{1} \tilde{\chi}(\xi)\left[\frac{1}{\xi-x}+Z(x, \xi)\right] d \xi+K_{0}(x)=\tilde{r}(x), x \in I_{1} \\
\quad \int_{-1}^{1} \tilde{\psi}_{1}(\xi) \frac{1}{\xi-x} d \xi+K_{1}(x)=0, x \in I_{1} \\
\int_{-1}^{1} \tilde{\psi}_{2}(\xi) \frac{1}{\xi-x} d \xi+K_{2}(x)=0, x \in I_{1}
\end{array}\right.
$$

here

$$
\tilde{\chi}(\xi)=\chi^{\prime}\left(\frac{a(\xi+1)}{2}\right), \tilde{\psi}_{i}(\xi)=\psi_{i}^{\prime}\left(\frac{\left(c_{1}-c_{0}\right) \xi+\left(c_{1}+c_{0}\right)}{2}\right), i=1,2
$$

$K_{i}(x)=\int_{-1}^{1} \tilde{\chi}(\xi) \tilde{f}_{i}(\xi, x) d \xi+\int_{-1}^{1} \tilde{\psi}_{1}(\xi) \tilde{R}_{i, 1}(x, \xi) d \xi+\int_{-1}^{1} \tilde{\psi}_{2}(\xi) \tilde{R}_{i, 2}(x, \xi) d \xi, i=0,1,2$

$\tilde{f}_{i}(\xi, x), \tilde{R}_{i, 1}(x, \xi), \tilde{R}_{i, 2}(x, \xi), \tilde{r}(x), i=0,1,2$ are known regular functions,

$\mathrm{Z}(x, \xi)=h_{1}\left(\frac{1}{\xi+x-2}+\frac{1}{\xi+x+2}\right)+h_{2}\left(\frac{x-1}{(\xi+x-2)^{2}}+\frac{x+1}{(\xi+x+2)^{2}}\right)+h_{3}\left(\frac{(\xi-1)(x-1)}{(\xi+x-2)^{3}}+\frac{(\xi+1)(x+1)}{(\xi+x+2)^{3}}\right)$

$h_{1}=-\frac{\kappa^{2}-3}{2 \kappa}, h_{2}=-\frac{2}{\kappa}, h_{3}=\frac{4}{\kappa}$.

The first equation in (6) contains two fixed singularities, so the special generalized method [22] was applied for its solving. The corresponding characteristic equation for the first equation in (6) was constructed. It is equal to the characteristic equation for the problem of an infinity wedge when the angle of openness is pi/2 [23].

According to the generalized method [22] the function $\tilde{\chi}(\xi)$ is expanded by series

$$
\tilde{\chi}(\xi)=\sum_{k=0}^{N-1}\left[s_{k}^{0} \rho_{k}^{-}(\xi)+s_{k+N}^{0} \rho_{k}^{+}(\xi)\right], \quad \xi \in[-1 ; 1]
$$

where $\begin{aligned} & \rho_{2 k}^{\mp}(\xi)=(1 \pm \xi)^{\operatorname{Re} \lambda_{k}} \cdot \cos \left(\operatorname{Im} \lambda_{k} \ln (1 \pm \xi)\right), \\ & \rho_{2 k+1}^{\mp}(\xi)=(1 \pm \xi)^{\operatorname{Re} \lambda_{k}} \cdot \sin \left(\operatorname{Im} \lambda_{k} \ln (1 \pm \xi)\right),\end{aligned} k=\overline{0, N-1}$.

The unknown functions $\tilde{\psi}_{1}(\xi), \tilde{\psi}_{2}(\xi)$ are expanded in the series by the Chebyshev polynomials of the second type [24]

$$
\tilde{\psi}_{i}(\xi)=\sum_{k=0}^{2 N-1} s_{k}^{i} \sqrt{1-\xi^{2}} U_{k}(\xi), \quad \xi \in[-1 ; 1], i=1,2
$$

The expressions (7)-(8) are substituted in SSIE (6), and SSIE (6) is solved with the help of the collocation method.

\section{THE STRESS INTENSITY FACTORS}

SIF are calculated by the formulae [19], [24] 


$$
\begin{aligned}
& K_{I-}=\sum_{k=0}^{2 N-1} s_{k}^{2} \frac{\sqrt{\pi\left(c_{1}-c_{0}\right)}(n+1)(-1)^{k}}{\sqrt{2}}, K_{I+}=\sum_{k=0}^{2 N-1} s_{k}^{2} \frac{\sqrt{\pi\left(c_{1}-c_{0}\right)}(n+1)}{\sqrt{2}}, \\
& K_{I I-}=\sum_{k=0}^{2 N-1} s_{k}^{1} \frac{\sqrt{\pi\left(c_{1}-c_{0}\right)}(n+1)(-1)^{k}}{\sqrt{2}}, K_{I I+}=\sum_{k=0}^{2 N-1} s_{k}^{1} \frac{\sqrt{\pi\left(c_{1}-c_{0}\right)}(n+1)}{\sqrt{2}}
\end{aligned}
$$

\section{NUMERICAL RESULTS AND DISCUSSION}

The calculations were done for the elastic semi-strip $\left(G=61.2781955 \cdot 10^{9} \mathrm{~Pa}, \mu=0.33\right)$ with parameters $p(x)=1 \mathrm{~Pa}$, $a=10 \mathrm{~m}, B=a$.

The crack is symmetrically located inside the semi-strip, so $c_{0}=a-c_{1}$. The dynamics of SIF changing when the crack's size is increasing from $10 \% a$ to $90 \% a$ is presented on Fig. 2 . As it can be seen, SIF are increasing when the crack's size increases. The values of SIF $K_{I}$ are larger than the values of SIF $K_{I I}$. When the crack's size is more than $90 \% a$ the fixed singularities at the crack's tips have to be considered.
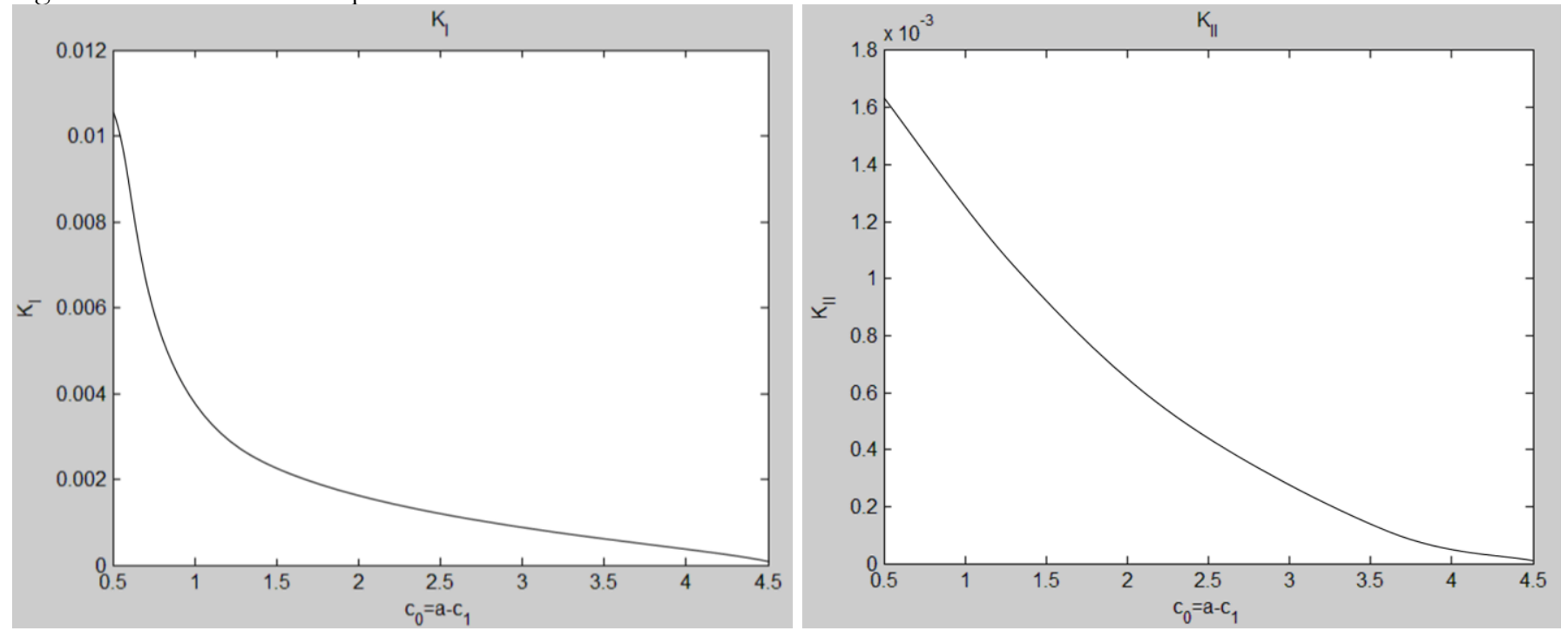

Figure 2: The changing of SIF when the crack's size is increasing.

\section{CONCLUSIONS}

1. The new methodic based on the apparatus of the matrix differential calculation and of the Green's function is proposed for the plane mixed problems of the semi-strip.

2. The generalized method was applied to consider the fixed singularities at the semi-strip's angle points.

3. The investigation of SIF was done, the limits of the proposed methodic were established.

\section{REFERENCES}

[1] Savruk, M.P., Osiv, P.N., Prokopchuk, I.V., Numerical analysis in plane problems of the crack's theory (in Russian), Naukova dumka, Kyiv, (1989).

[2] Morozov, N.F., Mathematical questions of the crack's theory (in Russian), Nauka, Moskow, (1984).

[3] Slepyan, L.I., Mechanics of cracks (in Russian), Sudnostroenie, L, (1990).

[4] Carpinteri, A., Spagnoli, A., Vantadori, S., Viappiani, D., Influence of the crack morphology on the fatigue crack growth rate: A continuously-kinked crack model based on fractals, Engineering Fracture Mechanics, 75 (2008) 579-589.

[5] Goldstein, R.V., Ryskov, I.N., Salganik, R.L., Central transverse crack in an infinite strip, Int. Journal of Fracture Mech., 6 (1970) 104-105. 
[6] Gecit, M.R., A cracked elastic strip bonded to a rigid support, International Journal of Fracture, 14, 6 (1978).

[7] Akbarov, S.D., Yahnioglu, N., Turan, A., Influence of initial stresses on stress intensity factors at crack tips in a composite strip, Mechanics of Composite Materials, 40, 4 (2004).

[8] Chai, H., A note on crack trajectory in an elastic strip bounded by rigid bustrates, International Journal of Fracture, 32 (1987) 211-213.

[9] Erdogan, F., Arin, K., A half plane and a strip an arbitrarily located crack, International Journal of Fracture, 11, 2 (1975).

[10] Lamzyuk, V.D., Mossakovshii, V.I., Sotnikova, S.D., On stresses in a strip with a crack, Journal of Mathematical Sciences, 70, 5 (1994).

[11] Kal'muk, L.I., Stashchuk, M.G., Pokhmurs'kii, V.I., Stress-intensity coefficients around the vertices of cracks and rigid inclusions in strips with clamped or free boundaries, Fiz.-Khim. Mekhanika Materialov, 26, 4 (1990) 65-75.

[12] Itou, H., Tani, A., A boundary value problem for an infinite elastic strip with a semi-infinite crack, Journal of Elasticity, 66 (2002) 193-206.

[13] Tian-You, F., Stress intensity factors of mode I and mode II for an infinite crack in a strip, International Journal of Fracture, 46 (1990) 11-16.

[14] Ballarini, R., Luo, H.A., Green's functions for dislocations in bonded strips and related crack problems, Int. Journ. of Fracture, 50 (1991) 239-262.

[15] Alexandrov, V.M., Pozharskii, D.A., To the problem of a crack on the elastic strip-half-plane interface, Mechanics of solids, 36, 1 (2001) 70-76.

[16] Chen, Yi-Zhou, Stress analysis for an infinite strip weakned by periodic cracks, Applied Mathematics and Mechanics, 25, 11 (2004).

[17] Civelek, M.B., Erdogan, F., Crack problems for a rectangular plate and an infinite strip, International Journal of Fracture, 19 (1982) 139-159.

[18] Dhaliwal, R.S., Singh, B.M., Two coplanar Griffith cracks in an infinitely long elastic strip, Journal of Elasticity, 11, 3 (1981).

[19] Popov, G.Ya., The elastic stress' concentration around dies, cuts, thin inclusions and reinforcements (in Russian), Nauka, Moskow, (1982).

[20] Vaysfel'd, N.D., Zhuravlova, Z.Yu., On one new approach to the solving of an elasticity mixed plane problem for the semi-strip, Acta Mechanica, 226, 12 (2015) 4159-4172. DOI: 10.1007/s00707-015-1452-x.

[21] Vaisfel'd, N.D., Zhuravlova, Z.Yu., Two-dimensional mixed problem of thermoelasticity for a semistrip, Journal of Mathematical Sciences, 228, 2 (2018) 105-121. DOI: 10.1007/s10958-017-3609-8.

[22] Vaysfel'd, N., Kryvyi, O., Zhuravlova, Z., On the stress investigation at the edge of the fixed elastic semi-strip, Frattura ed Integrità Strutturale, 38 (2016) 1-11. DOI: 10.3221/IGF-ESIS.38.01.

[23] Uflyand, Ya. S., Integral transformations in the problems of the elasticity theory (in Russian), Nauka, L., (1967).

[24] Zhuravlova, Z., Stress analysis near the tips of a transverse crack in an elastic semi-strip, Appl. Math. Mech. -Engl. Ed, (2017). DOI: 10.1007/s10483-017-2217-6. 